\title{
The impact of vitamin D deficiency and microbiome in psoriasis versus non immune-mediated diseases
}

\begin{abstract}
Psoriasis consists of a chronic inflammatory disease with systemic character and may be associated with several comorbidities. Although its etiopathogenesis has not yet been fully elucidated, it is known that it is an immune-mediated pathology, especially by $\mathrm{T}$ cells. Like other inflammatory and autoimmune disorders, psoriasis is also related to changes in the cutaneous microbiome. Vitamin D deficiency is one of the conditions associated with pathologies whose microbiome is altered, although the causal relationship between these events is not precisely determined. Thus, the objective of this study was to estimate the prevalence of vitamin D deficiency in patients with psoriasis who attended the Dermatology Clinic of ABC Medical School (FMABC) and compare it to the prevalence of vitamin D deficiency among patients with non-inflammatory pathologies. 88 patients with psoriasis and 91 patients with non-inflammatory diseases (Control Group) were evaluated and had their serum vitamin D measured. It was observed that the Psoriasis Group had lower serum vitamin D levels compared to the Control Group $(p<0.001)$ and It was observed that the chance of a person with psoriasis present low vitamin $\mathrm{D}$ levels is about seven times higher.
\end{abstract}

Volume 4 Issue 6 - 2020

Fernanda Soares Simoneti, Marisa Gonzaga
Cunha, Daniela Suzuki Locatelli, Fernando
Luiz Affonso Fonseca, Carlos D’Apparecida
Santos Machado Filho
Department of Dermatology, Faculdade de Medicina do ABC (FMABC), Brazil

Correspondence: Fernanda Soares Simoneti, Department of dermatology, Faculdade de Medicina do ABC (FMABC), Lauro Gomes, Brazil, Email nanda.simoneti@homail.com

Received: December 10, 2020| Published: December 23, 2020

Keywords: psoriasis, microbiome, autoimmunity

\section{Introduction}

Psoriasis is a chronic inflammatory disease, with a prevalence of approximately $1 \%$ in Brazil and Latin America and 3\% in Europe. Although skin involvement is widely known, including the nail apparatus, it is important to mention the systemic character of this condition, that may involve osteoarticular system and present a strong association with the metabolic syndrome or its components alone, and even greater cardiovascular risk. ${ }^{1}$

Clinically, psoriasis mayappear itself in various forms, being classified as follows: vulgar or plaque, guttate, inverted, palmoplantar, pustular, nail, erythrodermic and psoriatic arthritis. Within the pustular category, manifestations are subclassified as localized forms: palmoplantar pustulosis and acrodermatitis continua of Hallopeau; and generalized forms: von Zumbusch type (acute generalized pustular psoriasis) and impetigo herpetiformis, which occurs in pregnant women. ${ }^{1,2}$

The main histological findings in psoriasis are evident in the most superficial layers of the skin, where the inflammatory process leads to uncontrolled proliferation and altered keratinocyte differentiation. In addition, it also affects the vascular network present in the dermal layer and the interaction of keratinocytes with different cells of the innate and adaptive immune system. ${ }^{1}$

The cutaneous microbiome plays an active role in immune regulation and defense against pathogens, stimulating the production of antimicrobial peptides and the biofilm formation. The role of bacteria and virus in triggering the inflammatory cascade may be seen as an example the breakdown of immune tolerance involved in the etiopathogenesis of Crohn's disease, characterized by changes in the intestinal microbiome and classically associated with psoriasis. ${ }^{3-6}$ Beyond other autoimmune and chronic inflammatory pathologies, such as acne, rosacea, atopic dermatitis and vitiligo, psoriasis has also been associated with changes in the skin microbiome. There are reports describing the relative increase in Streptococcus and Staphylococcus aureus, reduction in Malassezia and Cutibacterium and also a decrease in immunoregulatory bacteria such as P. acnes and S. epidermidis, which could determine exacerbation of the Th17 axis. However, it remains uncertain whether the alteration of the cutaneous microbiome is the causal factor or the mere consequence of the inflammatory microenvironment. ${ }^{7-9}$

On the other hand, besides the regulatory action on calcium and phosphorus homeostasis classically described as the main function of Vitamin D, it has a wide range of effects, such as important role in cardiovascular protection, as an immunoregulator and even acting on the skin barrier and cell differentiation. ${ }^{1,10}$ In this context, the complex interaction among the alteration of the cutaneous microbiome, vitamin D levels and pathologies with a chronic autoimmune or inflammatory component, such as psoriasis, becomes evident due to mechanisms that are still uncertain.

The aim of this study was to estimate the prevalence of vitamin D deficiency in patients with psoriasis who attended the Dermatology Clinic of ABC Medical School (FMABC), compared to patients without inflammatory pathologies.

\section{Materials and methods}

The study was approved by the Ethics Committee of ABC Medical School (FMABC) and the data were collected only after agreement and voluntary signature of the Informed Consent Document by the selected patients. It was an observational, cross-sectional and retrospective study, with a quantitative approach, carried out at the Dermatology Discipline Clinic of FMABC, between the months of June and September 2019, with data obtained from medical records. Psoriasis patients were recruted by observing clinical manifestations, therapeutic history, and current treatment, as well as patients without inflammatory pathologies as a control group. Patients on current vitamin D replacement or less than a year ago were excluded.

The serum dosage of 25-hidroxyvitamin D [25(OH)D] was requested for both groups and the classification was established according to the reference values: deficiency (less than $20 \mathrm{mg} / \mathrm{dL}$ ), 
insufficiency (between 20 and $29 \mathrm{mg} / \mathrm{dL}$ ) and within the normal range (higher than $30 \mathrm{mg} / \mathrm{dL}$ ). The data obtained were organized in an Excel ${ }^{\circledR}$ electronic table and interpreted with Descriptive and Analytical Statistics (using the SPSS statistical software, version 15.0.1).

\section{Results}

A total of 196 individuals were included in the study, being 144 (73.4\%) female and $52(26.5 \%)$ male. The Psoriasis Group included 88 patients $(44.9 \%)$, with 53 female $(60.2 \%)$ and 35 male $(39.7 \%)$ patients. The individuals were classified according to age: up to 60 years (group 1), which represented $54.5 \%(\mathrm{n}=48)$, and over 60 years (group 2), which represented $45.4 \%(n=40)$ of the total. Psoriasis vulgaris was identified in 56 patients $(63.6 \%)$, psoriatic arthritis in 17 patients (19.3\%) and other forms of psoriasis (pustular, erythrodermic, palmoplantar, inverted and guttate) were found in 15 patients (17 $\%$ ), being regrouped in the category "Other Types of Psoriasis", in order to allow statistical analysis (Table 1). Regarding the therapeutic modality, $22.7 \%$ of patients $(\mathrm{n}=20)$ were using topical drugs, $57.9 \%$ $(\mathrm{n}=51)$ were using non-biological systemic drugs and $19.3 \%(\mathrm{~N}=17)$ were in systemic immunobiological therapy.

Table I Study description - Clinical manifestations in the Psoriasis Group

\begin{tabular}{lll}
\hline & Frequency & Porcentage \\
\hline Psoriasis vulgaris & 56 & $63,6 \%$ \\
Psoriatic arthritis & 17 & $19,3 \%$ \\
Other types of psoriasis & 15 & $17,0 \%$ \\
Total & 88 & 100,0 \\
\hline
\end{tabular}

There were 91 female (84.2\%) and 17 male patients (15.7\%) in the Control Group. Moreover, 82 patients were up to 60 years old $(75.9 \%)$ and 26 patients aged over 60 years $(24 \%)$.

The dosage of vitamin D in Psoriasis Group showed deficient or insufficient values in 76 patients (86.4\%). In addition, the dosages were also related to the type of psoriasis: 47 patients with psoriasis vulgaris $(83.9 \%), 13$ patients with other psoriasis manifestations $(86.6 \%)$ and 16 patients with psoriatic arthritis $(94.1 \%)$ had vitamin $\mathrm{D}$ values below normal. Therefore, low serum vitamin $\mathrm{D}$ levels were significantly more frequent in arthropathic form $(\mathrm{p}=0.06)$.

The Control Group had 52 patients (48.1\%) with insufficient or deficient serum vitamin D levels. In other words, it was observed that the Psoriasis Group had lower circulating vitamin D concentrations compared to the Control Group $(\mathrm{p}<0.001)$ and that the chance of a person with this disease having low vitamin $\mathrm{D}$ is about 7 times higher than a person without psoriasis.

Regarding therapeutic forms, vitamin D insufficiency or deficiency was observed in $18(90 \%)$ patients with topical treatment for psoriasis, $15(88.2 \%)$ of those undergoing systemic biological treatment and in $43(84.3 \%)$ patients undergoing non-biological systemic treatment. However, the difference in prevalence between them was not significant $(\mathrm{p}=0.8)$.

\section{Discussion}

Vitamin D plays a key role in inhibiting and modulating the immune system. It is able to prevent the pathological inflammatory response by suppressing inflammation mediated by Toll Like Receptors (TLR) in dendritic cell. Futhermore, it contributes to the maintenance of the dermoepidermal junction, through distribution of integrins, such as CD26 and ICAM-1, which are commonly altered in psoriasis.

Disorders in the response of the cutaneous immune system (innate and adaptive) are responsible for the development and maintenance of the inflammatory process of psoriasis. The kernel of the pathophysiology is the hyperactivation of the innate immune system, caused by certain triggers, culminating in the hyperproliferation of keratinocytes and production of cytokines and antimicrobial peptides in response to IL-22, IL-6, TNF- $\alpha$ and IFN $\gamma$. Although there is consensus on the fundamental role played by $\mathrm{T}$ lymphocytes, especially Th1, Th17 and Th22, in the complex etiopathogenesis of psoriasis, the participation of a series of triggers in the onset and aggravation of the condition remains nuclear. However it should be noted that in psoriasis, $\mathrm{T}$ lymphocytes play an important role, both in the production and activation of cytokines. ${ }^{11,12}$ Among other multiple factors mentioned in the literature, environmental factors stand out, such as alcohol, smoking, use of certain medications, trauma (Koebner phenomenon), infections and the participation of microorganisms acting as a trigger. ${ }^{13-16}$

On the other hand, literature associates vitamin $\mathrm{D}$ with the regulation of cutaneous immunity, by the suppression of proliferation of $\mathrm{T}$ cells, preferential development of auxiliary $\mathrm{T}$ cells (Th2), induction of regulatory cells, induction of activated B cell apoptosis, modulation in the activation of cytokines and dendritic cells. In the skin, vitamin D promotes differentiation and inactivates the proliferation of keratinocytes, selectively induces apoptosis, promotes the formation of a permeability barrier, regulates the immune system and induces the follicular cycle. This occurs through interaction between the activated metabolite 1,25-dihydroxyvitamin D3 [1,25 $(\mathrm{OH})$ 2D3] and the cell membranereceptor for vitamin D (VDR). ${ }^{17-23}$

Correale et al reported that vitamin D presents a greater immunomodulatory effect in women due to estrogen action. In this group, the inhibition of cells proliferation is greater, there is less production of INF- $\gamma$ and IL-17 and a greater amount of IL-10 secreting cells. ${ }^{24,25}$ Another relevant aspect involves the association between low vitamin D serum levels and autoimmune diseases, mediated by Th1 response, such as rheumatoid arthritis, type 1 diabetes, inflammatory bowel disease and multiple sclerosis, as described in a series of publications. ${ }^{26}$

The relationship between vitamin D levels and psoriasis has been extensively explored and documented in literature through last years, in order to establish a possible pathophysiological mechanism ${ }^{7-9,12,13}$ Bearing in mind that psoriasis is an autoimmune disease, mediated by Th1-Th17-Th22 response, it is possible that its manifestation consists, among other factors, consequence of vitamin D deficiency. ${ }^{24,25}$

In the present study, we observed that reduced levels of vitamin D were noticeably more frequent in the Psoriasis Group compared to the Control Group, with an estimated risk of having vitamin D deficiency or insufficiency seven times higher in patients with psoriasis. Psoriatic arthritis was more likely to be associated with reduced levels of vitamin $\mathrm{D}$, which supports the hypothesis that there is an interaction between this micronutrient and the arthropathic form. ${ }^{27}$

In general, insufficient levels have been attributed to several factors, including ethnicity, age, reduced sun exposure and reduced consumption of fish, eggs and liver. The minimum amount of vitamin $\mathrm{D}$ is hardly supplied by diet and the endogenous production from sunlight corresponds to $80-100 \%$ of the total amount needed. Sun protection, using sunscreen, hat and clothes that block UV rays, can 
contribute to reduction of the endogenous synthesis of vitamin D. In addition, the modern lifestyle with less time away from home or work further reduces daily sunlight exposure. ${ }^{17,18,28,29}$

The results obtained in this study can be justified in several ways, from disturbances in the hydration of the stratum corneum with reduced lipid content ${ }^{31,32}$ and the use of immunosuppressive drugs that interfere with the metabolism of vitamin $\mathrm{D}$, to the psychosocial repercussion of the disease in the patient with psoriasis, leading to less sunlight exposure precisely because of the habit of covering the affected body segments, which further decreases the synthesis of this vitamin in the organism. ${ }^{13,26}$

Vitamin D necessity can be suppressed in two ways: oral supplementation and skin synthesis through ultraviolet B (UVB) irradiation. $^{31,32}$ It is known that phototherapy with UVB light is able to activate vitamin D synthesis in keratinocytes, increasing its serum concentration, promoting activation of regulatory $\mathrm{T}$ cells. ${ }^{33-36}$ Furthermore, some antimicrobial peptides, including catelicidin, participate in adaptive and innate immune regulation, play an important role in controlling skin inflammation in patients with psoriasis and depend on UVB-induced vitamin D. ${ }^{37,38}$ In this regard, further studies are needed to assess the correlation between vitamin $\mathrm{D}$ levels and its possible interaction in therapeutic evolution. On the other hand, the use of vitamin D analogues in psoriasis topical treatment has been reported since the $1980 \mathrm{~s}$, demonstrating satisfactory results and comparable efficiency to potent corticosteroids..$^{39,40}$

\section{Conclusion}

Normal levels of vitamin D can prevent variations in immune homeostasis, modulate keratinocytes proliferation, regulate microbial flora and host's response to infectious diseases. In this context, the complex interaction - due to mechanisms that are still uncertain between alteration of the cutaneous microbiome, vitamin D levels and chronic pathologies with autoimmune or inflammatory component becomes evident.

Therefore, we can infer that the maintenance of serum vitamin D levels above $30 \mathrm{mg} / \mathrm{dL}$ could contribute to a better evolution when it comes to autoimmune and inflammatory diseases, such as psoriasis. Finally, serum dosage of 25-hydroxyvitamin D3, in addition to instituting careful vitamin replacement with laboratory control and periodic clinical evaluation is recommended.

\section{Conflicts of interest}

There are no conflicts of interest.

\section{Acknowledgment}

We are grateful to Dr. Karina G. Biagi (resident doctor of the dermatology department of FMABC), Simone M. Diniz and Julia A. Balthazar (medical students of FMABC) for their support and assistance throughout the execution of the study.

\section{Funding}

None.

\section{References}

1. Soleymani T, Hung T, Soung J. The role of vitamin D in psoriasis: a review. Int J Dermatol. 2015;54(4):383-392.

2. Mattozzi C, Paolino G, Richetta AG, et al. Psoriasis, vitamin D and the importance of the cutaneous barrier's integrity: An update. J Dermatol. 2016;43(5):507-514.
3. Chun RF, Adams JS, Hewison M. Back to the future: a new look at 'old' vitamin D. J Endocrinol. 2008;198(2):261-269.

4. Cantorna MT. Vitamin D and autoimmunity: is vitamin D status an environmental factor affecting autoimmune disease prevalence?Proc Soc Exp Biol Med. 2000;223(3):230-233.

5. Cai Y, Fleming C, Yan J. New insights of T cells in the pathogenesis of psoriasis. Cell Mol Immunol. 2012;9(4):302-309.

6. Savoia P, Novelli M, De Matteis A. et al. Effects of topical calcipotriol on the expression of adhesion molecules in psoriasis. J Cutan Pathol. 1998;25(2):89-94.

7. Carlo M, Giovanni Paolino, Antonio Giovanni R, et al. Psoriasis, vitamin $\mathrm{D}$ and the importance of the cutaneous barrier's integrity: An update. $J$ Dermatol. 2016;43(5):507-514.

8. Lewis DJ, Warren H Chan, Tiffany Hinojosa, et al. Mechanisms of microbial pathogenesis and the role of the skin microbiome in psoriasis: A review. Clin Dermatol. 2019;37(2):160-166.

9. Monteiro Júnior FC, Mandarino NR, et al. Deficiência de Vitamina D: um novo fator de risco cardiovascular?RevBrasCardiol. 2014;27(5):356365

10. Bell, D.S. et al. Protean manifestations of Vitamin D deficiency, part 1: the epidemic of deficiency. South Med J. 2011;104(5):331-334.

11. Sato-Deguchi E, Imafuku S, Chou B. et al. Topical Vitamin D3 analogues induce thymic stromal lymphopoietin and cathelicidin in psoriatic skin lesions. Br J Dermatol. 2012;167(1):77-84.

12. Boehncke WH, Dressel D, Zollner TM. et al. Pulling the trigger on psoriasis. Nature. 1996;379:777.

13. Bertrand KA, Edward Giovannucci, Yan Liu, Susan Malspeis, et al. Determinants of plasma 25- hydroxyvitamin D and development of prediction models in three US cohorts. Br J Nutr. 2012;108(10):18891896.

14. Lowes, M.A. et al. Immunology of Psoriasis. Annu. RevImmunol. 2014;32:227-255.

15. Lee YH, Song GG. Association between circulating 25-hydroxyvitamin D levels and psoriasis, and correlation with disease severity: a metaanalysis. Clin Exp Dermatol. 2018;43(5):529-535.

16. Rendon A, Schäkel K. Psoriasis Pathogenesis and Treatment. Int J Mol Sci. 2019;20(6):1475.

17. Lehmann B, Querings K, Reichrath J. Vitamin D and skin: new aspects for dermatology. Exp Dermatol. 2004;13:11-15.

18. Holick MF. Vitamin D deficiency. N Engl J Med. 2007;357:266-281.

19. Bikle DD. Vitamin D metabolism and function in the skin. Mol Cell Endocrinol. 2011;347(1-2):80-89.

20. Bell NH, Greene A, Epstein S, et al. Evidence for the alteration of the Vitamin D-endocrine system in blacks. J Clin Invest. 1985;76(2):470473.

21. El-Domyati M, Barakat M, Abdel-Razek R. et al. Apoptosis, P53 and Bcl-2 expression in response to topical calcipotriol therapy for psoriasis. Int J Dermatol. 2007;46(5):468-474.

22. Ryan C, Moran B, Mc Kenna MJ. et al. The effect of narrowband UV-B treatment for psoriasis on Vitamin D status during wintertime in Ireland. Arch Dermatol. 2010;146(8):836-842.

23. Werner de Castro GR, Neves FS, Pereira IA, et al. Resolution of adalimumab-induced psoriasis after Vitamin D deficiency treatment. Rheumatol Int. 2012;32(5):1313-1316.

24. Dankers W, Colin EM, van Hamburg JP, et al. Vitamin D in autoimmunity: molecular mechanisms and therapeutic potential. Front Immunol. 2017;7:697. 
25. Chun RF, Adams JS, Hewison M. Back to the future: a new look at 'old' vitamin D. J Endocrinol. 2008;198(2):261-269.

26. Cantorna, M.T. Vitamin D and autoimmunity: is vitamin D status an environmental factor affecting autoimmune disease prevalence?Proc Soc Exp Biol Med. 2000;223(3):230-233.

27. Filori A, Vestita M, Congedo M, et al. Association between psoriasis and vitamin D: Duration of disease correlates with decreased vitamin D serum levels: An observational case-control study. Medicine. 2018;97(25):e11185.

28. Reichrath J, Nurnberg B. Cutaneous Vitamin D synthesis versus skin cancer development. Dermato Endocrinology. 2009;1(5):253-261.

29. Wulf HC.The relation between skin disorders and Vitamin D. $\mathrm{Br} J$ Dermatol. 2012;166(3):471-472.

30. Darlenski, Evgeniya H, Ufuk A, et al. Epidermal Barrier and Oxidative Stress Parameters Improve During in 311 Nm Narrow Band UVB Phototherapy of Plaque Type Psoriasis. J Dermatol Sci. 2018;91(1):2834.

31. Rim JH, S J Jo, J Y Park, et al. Electrical measurement of moisturizing effect on skin hydration and barrier function in psoriasis patients. Clin Exp Dermatol. 2005;30(4):409-413.

32. Savoia P, Novelli M, De Matteis A. et al. Effects of topical calcipotriol on the expression of adhesion molecules in psoriasis. J Cutan Pathol. 1998;25(2):89-94.

33. Rácz E, Prens EP, Kurek D, et al. Effective treatment of psoriasis with narrow-band UVB phototherapy is linked to suppression of the IFN and Th17 pathways. $J$ Invest Dermatol. 2011;131(7):1547-1558.
34. Lehmann B, Knuschke P, Meurer M. The UVB-induced synthesis of vitamin D3 and 1 $\alpha, 25$-dihydroxyvitamin D3 (calcitriol) in organotypic cultures of keratinocytes: Effec-tiveness of the narrowband Philips TL01 lamp (311nm). Steroid BiochemMolec Biol. 2007;103(3-5):682-685.

35. Milliken SV, Wassall H, Lewis BJ, et al. Effects of ultraviolet light on human serum 25-hydroxyvitamin D and systemic immune function. $J$ Allergy Clin Immunol. 2012;129(6):1554-1561.

36. Hollox EJ, Huffmeier U, Zeeuwen PL, et al. Psoriasis is associated with increased beta-defensin genomic copy number. Nat Genet. 2008;40(1):23-25

37. Dombrowski Y, Peric M, Koglin S, et al. Cytosolic DNA triggers in ammasome activation in keratinocytes in psoriatic lesions. Sci Trans Med. 2011;3:82ra38.

38. Schauber J, Dorschner RA, Coda, AB, et al. Injury enhances TLR2 function and antimicrobial peptide expression through a vitamin Ddependent mechanism. J Clin Invest. 2007;117(3):803-811.

39. Lourencetti M, Abreu MM. Use of active metabolites of vitamin D orally for the treatment of psoriasis. Rev Assoc Med Bras. 2018;64(7):643-648.

40. Finamor DC, Rita Sinigaglia-Coimbra, Luiz C M Neves, et al.A pilot study assessing the effect of prolonged administration of high daily doses of vitamin D on the clinical course of vitiligo and psoriasis Dermatoendocrinol. 2013;5(1):222-234. 